\title{
Radiofrequency and Cryoablation Therapies for Supraventricular Arrhythmias in the Young: Five-Year Review of Efficacies
}

\author{
SUJATHA BUDDHE, M.D., HARINDER SINGH, M.D., WEI DU, PH.D., \\ AND PETER P. KARPAWICH, M.Sc., M.D. \\ From the Section of Pediatric Cardiology, Carmen and Ann Adams Department of Pediatrics, The Children's \\ Hospital of Michigan, Wayne State University School of Medicine, Detroit, Michigan
}

Background: Cryoablation (Cryo) has augmented radiofrequency $(R F)$ as the ablation energy choice for most supraventricular tachycardias (SVT). Although initial acute results and more recent, but limited, 3-36-month follow-up studies have been reported, more longer follow-up information is required to determine actual efficacy.

Methods: Data from patients with structurally normal hearts who underwent reentrant forms of SVT ablation at our institution from January 2005 to December 2009 were reviewed. These included demographics, clinical and electrophysiologic findings, and ablative energies used. Following apparent acute success, all patients were then reevaluated for any potential recurrences of SVT or preexcitation up to 5 years later.

Results: A total of 155 patients (83 male) were reviewed (mean age $13.4 \pm 3.7$ years). Ablations were predominantly right-sided (75\%). Atrioventricular reciprocating tachycardia was seen in $74 \%$ and atrioventricular node reciprocating tachycardia (AVNRT) in 17\% of patients. For concerns of atrioventricular node integrity, Cryo $\pm R F$ was user-preferred for anteroseptal accessory fiber locations and AVNRT. Acute success rate was $98 \%$ and chronic $83.2 \%$ over the next 5 years. Among patients with accessory pathways, recurrence was pathway number and location dependent: significantly higher $(P<$ $0.05)$ if they were right anterior-anteroseptal, multiple, or with a broad-distribution pattern. There were no significant differences in recurrence rates with use of RF or its combination with Cryo.

Conclusion: Radiofrequency ablation and Cryo are both effective therapies for pediatric patients. Although use of Cryo with RF in combination may enhance safety while affording comparable success, risk of recurrence still persists in the current era among patients depending on accessory pathways connection location and characteristics. (PACE 2012; 35:711-717)

\section{supraventricular tachycardia, ablation, radiofrequency, cryotherapy, recurrence}

\section{Introduction}

Radiofrequency catheter ablation (RFA) has revolutionized the treatment of diverse supraventricular tachycardias (SVT) in children. ${ }^{1}$ Although developed in the 1980s and widely applied in the 1990s, formalized guidelines for its use in clinical practice were not developed until recently. ${ }^{2}$ Based on data from the Pediatric Radiofrequency Catheter Ablation Registry, acute failure rates fell

Conflict of interest: None.

Disclosures: None.

Address for reprints: Peter P. Karpawich, M.Sc., M.D., Director, Cardiac Electrophysiology, Pediatric Cardiology, The Children's Hospital of Michigan, 3901 Beaubien Blvd, Detroit, MI 48201. Fax: 313-993-0894; e-mail: pkarpawi@dmc.org

Received February 11, 2011; revised January 10, 2012; accepted January 24, 2012.

doi: 10.1111/j.1540-8159.2012.03372.x from $9.6 \%$ in the early 1990 s to $4.3 \%$ at the end of the decade. ${ }^{3}$

RFA, however, can cause complications including thromboembolic events resulting in neurological damage, coronary artery injury, atrioventricular (AV) node injury, conduction block, ${ }^{4}$ and even death. ${ }^{5-7}$ Cryoablation (Cryo) is a newer energy modality of catheter ablation which has proven to be safe in many studies. ${ }^{8,9}$ Previous studies have reported acute success rates of only $69-92 \%$ which is less than that reported for $\mathrm{RFA}^{10,11}$ and higher arrhythmia recurrence compared to RFA. ${ }^{8}$

Most of the initial RFA and Cryo studies reported only acute success results. More recent but limited combined RFA/Cryo studies have reported from 9 to $35 \%$ recurrence rates. ${ }^{12-14}$ But, there is a paucity of studies comparing early and late ablative success rates using RFA or Cryo or combinations of both in pediatric patients with structurally normal hearts. We sought to identify potential risk factors for SVT recurrence after initial successful ablation in the current era.

(C)2012, The Authors. Journal compilation @2012 Wiley Periodicals, Inc. 


\section{Methodology}

This was a retrospective study conducted at The Children's Hospital of Michigan, Division of Cardiology. The study was approved by the institutional review committee at Wayne State University School of Medicine, Detroit Medical Center. The inclusion criteria were patients with reentrant forms of SVT with documented evidence by electrocardiogram (EKG), Holter, or event monitor referred for an initial catheter ablation. Patients with repeat procedures were excluded. To allow for a degree of consistency in comparison of energy sources, per se, patients with structural congenital heart defects or previous ablations were excluded. As such, only patients with atrioventricular node reciprocating tachycardia (AVNRT), atrioventricular reciprocating tachycardia (AVRT), sinus reentry, as well as His bundle reentrant forms of SVT were included in this study. Also, since the purpose was to evaluate energy delivery efficacy in discrete select regions in deference to performance of a more extensive linear "maze," patients with primary atrial muscle tachycardias such as flutter/fibrillation also were not included. Ectopic atrial tachycardia was not included as it was considered to be an automatic tachycardia.

All clinical, intracardiac electrophysiological study (EPS) and follow-up data were reviewed. The demographic and clinical data collected included patient's age, gender, ethnicity, height, weight, body surface area, clinical manifestations, as well as presence or absence of preexcitation at rest and on exercise stress testing. EPS data included site and number of locations of accessory pathways (APs), and use of RFA or Cryo or both. Follow-up data at 1-2 weeks and from 3 to 77 months later included recurrence of symptoms; results of treadmill exercise stress testing; and 15-lead EKG, Holter, or telephone event monitor reports and any repeat EPS procedures.

All patients underwent EPS under the same protocol by two established pediatric electrophysiologists. All procedures were performed under standard general anesthesia. Established techniques using multielectrode catheter placement for intracardiac pacing and mapping were performed in all cases. A standard flexible quadripolar roving electrode catheter was used for selective AP site mapping as required. A threedimensional mapping system (St. Jude Medical, Minnetonka, MN, USA) was used to define anatomical and electrical landmarks to limit fluoroscopic imaging support for all patients post mid-2007.

Among patients with overt preexcitation standard mapping for prograde AP conduction to delineate location was conducted prior to initiation of stimulation protocols. The region of electrical interest was established to include atrial and ventricular activation. For this study, the now standard, albeit somewhat arbitrary, AP site map was used to define general AP locations, with the realization that AP connections are not necessarily discrete and separate as the AV annulus has limited definable boundaries. ${ }^{3,15}$ Multiple pathways were defined by established techniques of catheter mapping 1-3 cm apart or change in any preexcited QRS morphology following ablation at different sites or induction of another form of tachycardia with a different QRS morphology or axis. ${ }^{18}$ Since fluoroscopic mapping-based identification of presence of multiple APs is inexact, patients in whom mapping of AP connections and ablations were performed across several or adjoining traditional fluoroscopic locations (e.g., anterior-anteroseptal) were said to have broad-distribution fibers, ostensibly with wide atrial and ventricular insertions, in deference to more discrete and, by inference, compact APs. ${ }^{19}$

Standard programmed electrical stimulation (PES) protocols were instituted specifically to only induce tachycardia, not to induce nonclinical atrial flutter/fibrillation (AF). These included single, double, and burst atrial extra stimuli at standard variable drive-paced cycle lengths (all $>200 \mathrm{~ms}$ ), both with and without isoproterenol infusion $(0.1 \mathrm{mcg} / \mathrm{min})$, as required for arrhythmia induction. Once the SVT mechanism (e.g., AVRT or AVNRT) was identified and any APs located by standard catheter mapping, RFA and/or Cryo ablations were performed. As part of the established EPS protocol at our institution, risk stratification of each patient with evaluation of refractory periods, inducibility of arrhythmias, and concomitantly measured hemodynamics, especially among patients with overt preexcitation and potential for sudden death, was performed. This also permitted a more accurate determination of "success" as the entire EPS stimulation protocol was repeated following ablation. In instances of induced hemodynamic instability, cardioversion was performed.

Each RFA application was performed up to 60 seconds to attain a tissue temperature from $50^{\circ} \mathrm{C}-70^{\circ} \mathrm{C}$ (Boston Scientific, Natick, MA, USA) and each Cryo was performed using a 4-mm Cryo catheter for up to 4 minutes using the two-tiered approach of initial tissue chilling at $-30^{\circ} \mathrm{C}$ for several seconds followed by cellular disruption achieved at a temperature of $-70^{\circ} \mathrm{C}$ (CryoCath, Medtronic). Once tachycardia occurred, any spontaneous deterioration to AF was recorded. All patients were observed for 60 minutes following apparent successful ablation. This was followed by 
a full repeat PES with and without isoproterenol to evaluate inability to reinduce any SVT, as well as administration of adenosine (for AVRT cases) to confirm evidence of any residual accessory orthodromic connections. Recurrence required additional ablation. Success was therefore defined as meeting several criteria following a 60-minute postablation waiting interval: (1) A normal QRS in all patients and no visible preexcitation among those patients with previous preexcitation; (2) Inability to reinduce SVT in spite of repeat PES with and without isoproterenol infusion among all patients; (3) No evidence of residual preexcitation following adenosine administration. Retrograde VA conduction, which can be a common ancillary finding in children, was not uniformly tested in all patients postablation and therefore not included as a success criteria per se.

After the procedure, as part of hospital policy, patients were observed overnight and a standard 15-lead EKG to reconfirm a normal QRS and echocardiogram/Doppler to evaluate valve integrity were performed the next day prior to discharge. All patients were then reevaluated from 1 to 2 weeks followed by 1-3 months later and then on a recommended yearly basis for any SVT recurrences. All follow-up clinical evaluations included a standard 15-lead EKG. If patients had any recurrence of clinical symptoms, telephone event and/or Holter monitor recording equipment was provided for confirmation depending on the discretion of the cardiologist. For SVT recurrence, medical management was initiated before repeating the electrophysiology (EP) procedure. For statistical analysis, patients were divided into two groups: Group 1, those with SVT recurrences and Group 2, those without SVT recurrences. Group 1 patients also included those with a return of preablation preexcitation with or without SVT.

\section{Statistics}

This was a retrospective study. All data were reported as mean and standard deviation for continuous variables and frequency for categorical variables. The two groups were compared using independent sample $t$-test, Mann-Whitney U test, Fisher's exact or $\chi^{2}$ tests depending on the distribution of the data. All statistical analyses were performed using SAS software version 9.1 (SAS Institute, Cary, NC, USA).

\section{Results}

A total of 155 patients with structurally normal hearts underwent catheter ablation at our institution for the indicated forms of SVT from January 2005 to December 2009. Of these, 83 were males and 72 were females. Mean age at catheterization was $13.4 \pm 3.7$ years. Based on listed ethnicity, 99 (64\%) were of European-, 34 $(22 \%)$ African-, five (3\%) Mid-Eastern-, and one (1\%) Asian-American ancestries.

Of the initial 155 patients, acute success was achieved in 153 (98\%). Later, 24 patients $(16.8 \%)$ developed recurrence of SVT or overt preexcitation at a mean follow-up interval of 38 months (Group 1), whereas 129 had no evidence of either recurrence (Group 2). Median follow-up interval was 41 months (1-77 months) and 21 patients $(13.5 \%)$ were lost to followup. Of the remaining 134 patients not lost to follow-up, clinical information was available on 108 patients $(81 \%)$ at 2 years, $82(61 \%)$ at 3 years, $52(39 \%)$ at 4 years, and $29(22 \%)$ at 5 years postablation. Although patients in the recurrence group were younger than those in the nonrecurrence group $(\mathrm{P}<0.05)$, the other demographic distributions including weight, sex, and race were not significantly different between both groups (Table I). Pre-EPS, overt preexcitation on resting EKG was seen in 82 patients $(53 \%)$. This included 13 (50\%) in Group 1 and 69 (54\%) in Group 2. This was not significantly different $(\mathrm{P}=0.8)$. Tachycardia types were typical for a pediatric population: $74 \%$ atrioventricular reentry (AVRT) with accessory bypass fibers, 17\% "slowfast" AV node (AVNRT), $5 \%$ sinus node, and $4 \%$ His bundle reentry.

Fifty-five patients $(36 \%)$ had more than one accessory connection catheter map location including $58 \%$ of Group 1 and $31 \%$ of Group 2 $(\mathrm{P}=0.01)$. Among these patients, multiple accessory connection locations encompassing both right and left valve locations were found

\section{Table I.}

Patient Demographics between Groups

\begin{tabular}{lccc}
\hline & $\begin{array}{c}\text { Recurrence } \\
(\mathbf{n}=\mathbf{2 6})\end{array}$ & $\begin{array}{c}\text { No-recurrence } \\
(\mathbf{n}=\mathbf{1 2 9})\end{array}$ & P- value \\
\hline Age & $11.7 \pm 3.6$ & $13.6 \pm 3.6$ & $<0.05$ \\
Weight & $54 \pm 21$ & $53 \pm 22$ & 0.9 \\
Sex-Male & $10(39 \%)$ & $73(57 \%)$ & 0.1 \\
European & $17(65 \%)$ & $82(64 \%)$ & 0.9 \\
$\quad$ ancestry & & & \\
African ancestry & $7(27 \%)$ & $27(21 \%)$ & 0.6 \\
Mid-Eastern & $1(4 \%)$ & $4(3 \%)$ & 1.0 \\
$\quad$ ancestry & & & \\
\hline
\end{tabular}

No significant difference between the two groups in terms of gender, weight, and ethnicity. Patient age, however, was significant. There was only one patient of Asian ancestry. That patient had no recurrence.

Value expressed as mean \pm standard deviation or as number (percentage). 
in $10 \%$ whereas broad-distribution connections were found in $26 \%$. Of these latter, the most common was an overlap between right anterior and anteroseptal catheter-mapped regions. The average number of mapped AP locations in Group 1 was $1.7 \pm 0.7$ and $1.4 \pm 0.6$ in Group $2(P<0.05)$.

Ablations were required in variable areas depending on accessory fiber locations or mechanism of SVT. In this review, AVNRT was considered a right-sided tachycardia which contributed to the difference between right and left locations. Therefore, ablations were predominantly performed on the right side: 112 patients $(75 \%)$ versus left side in 54 patients (25\%). Among all patients with right-sided tachycardia foci, anteroseptal locations were seen in 42 patients $(20 \%)$, anterior in $33(15 \%)$, and midseptal in $30(14 \%)$; AVNRT was seen in $35(17 \%)$, sinus node reentry in $11(5 \%)$, and His bundle in five $(4 \%)$. AVNRT ablations were performed to either eliminate or modify "slow" AV pathway conduction properties.

Among AVRT mechanisms, SVT recurrence was significantly higher among patients following right anteroseptal fiber ablation with a 33\% recurrence rate $(\mathrm{P}<0.01$ between both groups $)$ and an odds ratio of 4.2. Recurrence was not significantly higher in the other fiber locations, including AVNRT (Table II). However, among patients with multiple or broad-distribution AP distributions, $27 \%$ had recurrences $(\mathrm{P}=0.01$ between both groups) and an odds ratio of 3.0.

During the EPS, RFA and/or Cryo were used depending on the location of the APs and at the discretion of the electrophysiologist performing the procedure. With concern for AV

Table II.

Accessory Fiber Locations/Ablation Sites between Groups

\begin{tabular}{lrcc}
\hline Fiber Locations & $\begin{array}{c}\text { Recurrence No-recurrence } \\
(\mathbf{n}=\mathbf{4 2})\end{array}$ & $\begin{array}{l}(\mathbf{n}=\mathbf{1 7 3}) \\
\end{array}$ & P-value \\
\hline Right anteroseptal & $14(33)$ & $28(16)$ & $<0.01$ \\
Right anterior & $8(19)$ & $25(15)$ & 0.1 \\
Midseptal & $4(10)$ & $26(15)$ & 0.3 \\
Left posterolateral & $6(14)$ & $48(28)$ & 0.2 \\
AVNRT & $5(12)$ & $30(17)$ & 0.8 \\
Sinus reentry & $2(5)$ & $9(5)$ & 1.0 \\
His bundle & $3(7)$ & $7(4)$ & 0.2 \\
\hline
\end{tabular}

Recurrence is significantly higher in patients with right anteroseptal fiber locations $(P<0.01)$. No other fiber locations were associated with increased recurrence.

Value expressed in number (percentage). node integrity, RFA and Cryo were preferably used for AP locations away from and in close proximity to the normal conduction pathway, respectively. For most of the fiber locations close to the conduction pathway, like AVNRT, Cryo was used first for cryomapping and cryoablation. RFA was then used in the same location when it was deemed safe by cryomapping or for placing linear lesions further away from the conduction pathway. Patients were divided into three groups based on type of energy source used for ablation. RFA alone was used in 87 patients (107 fiber locations), Cryo alone in nine patients (11 fiber locations), and both RFA/Cryo were used in 59 patients (97 fiber locations). Cryo with/without RFA was preferably used for AVNRT and right anteroseptal fibers to optimize avoidance of AVN conduction damage. RFA alone was preferred for left-sided fibers. When required due to fiber location, coronary sinus RFA was also performed (Table III). The number of RFA ablations applied was higher in the RFA + Cryo group compared to RFA group, indicating that this group required more ablations to achieve success. The number of Cryo, though higher in RFA + Cryo group compared to Cryo alone, did not reach statistical significance. Electroanatomic 3D mapping was used for all patients post mid-2007. However, comparison on recurrence cannot be made between those patients with and without 3D mapping as the follow-up period was significantly higher for patients without 3D mapping compared to those in whom it was used, resulting in higher recurrences in the pre-2007 group without use of 3D mapping.

There were 34 African-American patients forming $22 \%$ of the study group, including 16 males (47\%). Of these, $22(65 \%)$ had overt preexcitation. Seventeen (50\%) of them exhibited multiple or broad-distribution right-sided AP connections compared to only $31 \%$ in the nonAfrican-American races. This difference was significant $(\mathrm{P}=0.04)$. A total of 51 AP fiber locations were identified among this ethnic group, including 40 (78\%) right-sided and $11(22 \%)$ leftsided fibers. Among the right-sided APs, right anterior were seen in $12(30 \%)$, right anteroseptal in eight $(20 \%)$, and midseptal in nine $(23 \%)$. AVNRT was seen in seven $(17 \%)$, sinus reentry in two (5\%), and His reentry in two (5\%).

Acute success rates as defined earlier (inability to reinduce any arrhythmia, no preexcitation with adenosine, and a normal QRS morphology) was $98 \%$. Both Cryo with/without RFA Groups had $100 \%$ acute success. Of the two patients in the RFA-only Group who had acute failure, one had both right anterior and anteroseptal fibers and the other had left posterolateral fibers. Both patients 
Table III.

Accessory Fiber Locations between Groups Based on Type of Energy Source Used for Ablation

\begin{tabular}{lcccc}
\hline Fiber Locations & RFA $(\mathbf{n}=\mathbf{1 0 7})$ & Cryo $(\mathbf{n}=\mathbf{1 1})$ & RFA + Cryo $(\mathbf{n}=\mathbf{9 7})$ & P-value \\
\hline Number of patients & 87 & 9 & 59 & \\
Right anteroseptal & $13(12)$ & $3(27)$ & $26(27)$ & $<0.01$ \\
Right anterior & $16(15)$ & $0(0)$ & $17(18)$ & 0.1 \\
Midseptal & $14(13)$ & $0(0)$ & $16(16)$ & 0.8 \\
Left posterolateral & $44(42)$ & $0(0)$ & $10(10)$ & $<0.01$ \\
AVNRT & $10(9)$ & $5(46)$ & $20(21)$ & $<0.01$ \\
Sinus reentry & $8(7)$ & $1(9)$ & $2(2)$ & 0.2 \\
His bundle & $2(2)$ & $2(18)$ & $21 \pm 16$ & 0.36 \\
Number of RFA & $13 \pm 11$ & - & $11 \pm 8$ & 0.01 \\
Number of Cryo & - & $7 \pm 5$ & & 0.5 \\
\hline
\end{tabular}

Cryo with or without RFA was preferably used for right anteroseptal fiber locations and AVNRT while RFA alone was preferably used for left-sided fiber locations.

P-value comparing RFA group with RFA + Cryo group.

Value expressed in number (percentage) or mean \pm standard deviation.

had persistent preexcitation after the ablation, but no tachycardia could be induced with PES after waiting for an hour and parents deciding against further ablations. Following initial success, 16.8\% of patients had SVT/preexcitation recurrences during the follow-up interval. Recurrence risk was not significantly different with use of RFA (14\%), Cryo (22\%), or both (20\%). By survival analysis, no significant difference in recurrence was found between the three groups (Fig. 1). The EPS were performed by two electrophysiologists in our institution and the recurrence risk was not significantly different between them. Using regression analysis, recurrence risk was highest in patients with right anteroseptal fibers.

Rhythm complications of flutter/fibrillation occurred in five patients during PES with rapid ventricular response and hemodynamic deterioration that required cardioversion. There were no AV conduction problems associated with any initial ablation. There was no evidence of coronary sinus or AV valve problems clinically or by ultrasound. There were no complications of stroke, coronary artery injury, and no deaths were encountered.

\section{Discussion}

SVT is a frequently encountered arrhythmia in pediatric patients. Medical therapy requires patient and family compliance which is often less than optimal and antiarrhythmic drugs can be proarrhythmic. Although surgical ablation was an available alternative previously, catheterdelivered RFA and Cryo have now revolutionalized treatment modalities. The safety and efficacy has been improving over the years with the

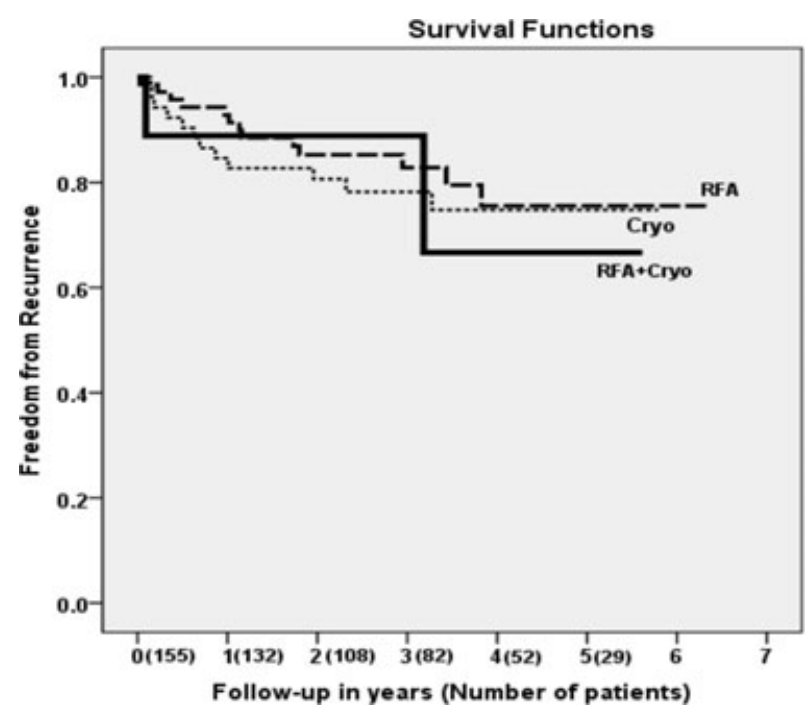

Figure 1. Kaplan-Meyer curve comparing freedom from recurrence between three groups based on type of energy source used. There is no significant difference in recurrence between the three groups.

learning curve phenomenon as applied to use in children. ${ }^{14,15}$

RFA is the widely used older energy source for ablation. The success has been improving over the years. The Pediatric Radiofrequency Catheter Ablation Registry reported an improvement in the initial success rates from $90.4 \%$ (1991-1995) to $95.2 \%$ (1996-1999) for RFA among all patients. ${ }^{3}$ Our report has a better acute procedural success rate for RFA at $98 \%$. Cryotherapy is the newer 
modality of treatment. An acute success rate of $87.1 \%$ for cryotherapy has been reported, which is less than that for RFA with success rates for AVNRT ablation higher than for AVRT $(95.5 \%$ vs $62.5 \%, \mathrm{P}<0.05) .{ }^{10}$ In our study, with Cryo alone, the acute success rate was $100 \%$, but only a limited number of patients were in this group. For many of our patients, RFA was used together with Cryo and the success rate was also $100 \%$. The reason for such a high acute success rate in our study reflects use of RFA along with Cryo in locations where RFA could be used safely.

Rates of initial success also depended on AP location. In our patient population, among those with AVRT, the fiber locations were predominantly right-sided (66\%). This is in contradistinction to the findings of the initial Prospective Assessment after Pediatric Catheter Ablation as well as a more recent study in which right-sided pathways were found in only $17-30 \%$ of patients. ${ }^{4,20}$ Although the "anatomical maps" do help to standardize ablation catheter locations by fluoroscopy, they are somewhat arbitrary and not based on any direct histological evidence. Previous studies have reported multiple AP fibers in $5-20 \%$ of patients based on clinical findings. ${ }^{20}$ However, right-sided AP connections are anatomically different from left. The associated folding of the atrium over the ventricle, inherent anatomical variability of the right-sided A-V ring, AP physical characteristics, and orientation relative to atrial and ventricular myocardium may all contribute to an inherent overlap among the sites, especially on the right. Wide AP branching and spatial separation of any two APs, as shown in histologic studies, may present with a clinical picture of multiple or broad-distribution connections, hindering effective catheter mapping and ablation. ${ }^{16}$ In the previous era of surgical ablation, an extensive valve annuloplasty was often performed to ensure AP interruption. ${ }^{17}$ In this regard, patients with such connections may be expected to have less optimal ablation results than those with more discrete connections. ${ }^{18,19}$

A comparison between our data and the previous reports mentioned earlier also shows an interesting discrepancy in patient ethnicity. Our database shows that $22 \%$ of our patients were of African-American ancestry compared to $3-7 \%$. There were more patients with broad-distribution or multiple fiber locations as noted earlier. Our data, therefore, suggest that ethnicity may be an important component of AP type and location and, by inference, tachycardia recurrence. Since there are regional ethnic population diversities across the United States, any one pediatric EP center may have limited exposure to any one ethnic group. In this regard, we agree with the recent report by Hsu et al. that ethnicity may be an important variable contributing to different reported AP locations, successes, and recurrences among different institutions. ${ }^{21}$ However, a larger multi-institutional/multi-ethnic population study will be required to further evaluate this concept.

We purposely used a very broad definition of "recurrence" to include both only recurrent preexcitation as well as bona fide tachycardia. In this regard, the late 5-year recurrence rate in our study was $16.8 \%$. Previous studies have reported recurrence rates varying from 4.7 to $45 \%$, with RFA at $4.7-8.2 \%^{12,13}$ and Cryo even higher at $13-45 \% .^{14,22}$ In our current study, no significant differences in recurrence rates were noted for use of RFA or RFA + Cryo (14 and $20 \%$ respectively, $\mathrm{P}=0.5$ ). The Cryo-only group had recurrence rate of $22 \%$ which was not significantly different compared to others, but as this group had very limited number of patients, this comparison may not be adequately powered. The use of RFA combined with Cryo could have improved the overall success rates in our study group. The recurrence rate also varied depending on substrate location. Although there has been improvement over the last two decades, the success rate for RFA ablation of right is less than for left-sided pathways, 90\% versus $98 \%{ }^{4}$ From the international registry, a $75 \%$ success rate was reported for right septal fibers. ${ }^{11}$ In our study, higher recurrence was noted for right-sided fibers with right anteroseptal fibers (33\%), right anterior (24\%), left-sided fibers (20\%), and AVNRT (14\%).

AV block has been reported to occur in $1.2 \%$ of patients following ablation, mostly for AVNRT and septal accessory pathways. ${ }^{4}$ Unlike RFA, Cryo has been associated with a decreased incidence of reported complications. It has been shown that cooling of the myocardium to $-30^{\circ} \mathrm{C}$ results in reversible tissue modification. ${ }^{23}$ This adds a theoretical benefit of predetermining any potential adverse problems prior to definitive cellular disruption once the temperature reaches $-70^{\circ} \mathrm{C}$. In animal studies, cryoablated sites were more homogeneous and showed viable myocytes with fibrotic tissue changes but no signs of chronic inflammation. ${ }^{24}$ Cryo has also been shown to have better catheter adherence and spares the endothelial lining leading to less thrombogenicity compared to RFA. ${ }^{25,26}$ Use of Cryo for fiber locations close to AV node and limiting RFA to safer regions could be the cause for the low incidence of complications in our study.

Unlike previous studies, we have reported long-term follow-up in pediatric patients. We have used RF with Cryo which is different compared to previous studies. Compared to previous studies, although our overall success rates seem to be 
similar, we had many patients with right-sided APs and we have higher success rates for these fibers. We thus propose that use of RFA with Cryo together would increase success rates compared to either energy source alone for those fiber locations that have traditionally been known to have poor ablation success.

\section{Limitations}

This was a single-center retrospective study with a relatively small number of patients in the Cryo-only group. Also, our patient demographics and accessory fiber locations differ from other studies making generalization of the results difficult. Further multicenter prospective studies, including ethnic diversity, will be needed to validate the findings.

\section{References}

1. Belhassen B, Rogowski O, Glick A, Viskin S, Ilan M, Rosso R, Eldar M. Radiofrequency ablation of accessory pathways: A 14 year experience at the Tel Aviv Medical Center in 508 patients. Isr Med Assoc J 2007; 9:265-270.

2. Blomstrom-Lundqvist C, Scheinman MM, Aliot EM, Alpert JS, Calkins H, Camm AJ, Campbell WB. ACC/AHA/ESC guidelines for the management of patients with supraventricular arrhythmiasExecutive summary. A report of the American College of Cardiology/American Heart Association Task Force on Practice Guidelines and the European Society of Cardiology Committee for Practice Guidelines (Writing Committee to Develop Guidelines for the Management of Patients with Supraventricular Arrhythmias) developed in collaboration with NASPE-Heart Rhythm Society. J Am Coll Cardiol 2003; 42:1493-1531.

3. Kugler JD, Danford DA, Houston KA, Felix G. Pediatric radiofrequency catheter ablation registry success, fluoroscopy time, and complication rate for supraventricular tachycardia: Comparison of early and recent eras. J Cardiovasc Electrophysiol 2002; 13:336-341.

4. Van Hare GF, Javitz H, Carmelli D, Saul JP, Tanel RE, Fischbach PS, Kanter RJ, et al. Prospective assessment after pediatric cardiac ablation: Demographics, medical profiles, and initial outcomes. J Cardiovasc Electrophysiol 2004; 15:759-770.

5. Cannon BC, Kertesz NJ, Friedman RA, Fenrich AL. Use of tissue plasminogen activator in a stroke after radiofrequency ablation of a left-sided accessory pathway. J Cardiovasc Electrophysiol 2001; 12:723-725.

6. Schaffer MS, Gow RM, Moak JP, Saul JP; Participating members of the Pediatric Electrophysiology Society. Mortality following radiofrequency catheter ablation (from the Pediatric Radiofrequency Ablation Registry). Am J Cardiol. 2000; 86:639-643.

7. Hope EJ, Haigney MC, Calkins H, Resar JR. Left main coronary thrombosis after radiofrequency ablation: Successful treatment with percutaneous transluminal angioplasty. Am Heart J. 1995; 129:1217-1219.

8. Gaita F, Haissaguerre M, Giustetto C, Grossi S, Caruzzo E, Bianchi F, Richiardi E, et al. Safety and efficacy of cryoablation of accessory pathways adjacent to the normal conduction system. J Cardiovasc Electrophysiol 2003; 14:825-829.

9. Drago F, De Santis A, Grutter G, Silvetti MS. Transvenous cryothermal catheter ablation of re-entry circuit located near the atrioventricular junction in pediatric patients: Efficacy, safety, and midterm follow-up. J Am Coll Cardiol 2005; 45:1096-1103.

10. Miyazaki A, Blaufox AD, Fairbrother DL, Saul JP. Cryo-ablation for septal tachycardia substrates in pediatric patients: Mid-term results. J Am Coll Cardiol 2005; 45:581-588.

11. Kirsh JA, Gross GJ, O’Connor S, Hamilton RM. Transcatheter cryoablation of tachyarrhythmias in children: Initial experience from an international registry. J Am Coll Cardiol 2005; 45:133-136.

12. Lee PC, Hwang B, Chen SA, Tai CG, Chen YJ, Chiang CE, Meng CC. The results of radiofrequency catheter ablation of supraventricular tachycardia in children. Pacing Clin Electrophysiol 2007; 30:655-661.

\section{Conclusion}

RFA and Cryo are safe and efficacious treatment options for pediatric patients with SVT. They are associated with excellent acute procedural success. But since first applied, chronic efficacy of ablation therapies in the young have not yet been well established. Our report demonstrates that in spite of apparent acute success, the risk of late recurrence, either as QRS preexcitation and/or arrhythmias, persists in the current era especially among patients with broaddistribution, multiple and those with anteroseptal accessory fiber locations. Use of Cryo with RF in combination may enhance safety while affording comparable success, especially for fiber locations that have been traditionally known to have higher recurrence rates.

13. Bae EJ, Ban JE, Lee JA, Jin SM, Noh CI, Choi JY, Yun YS. Pediatric radiofrequency catheter ablation: Results of initial 100 consecutive cases including congenital heart anomalies. J Korean Med Sci 2005; 20:740-746.

14. Gaita F, Montefusco A, Riccardi R, Scaglione M, Grossi S, Caponi D, Caruzzo E. Acute and long-term outcome of transvenous cryothermal catheter ablation of supraventricular arrhythmias involving the perinodal region. J Cardiovasc Med (Hagerstown) 2006; 7: 785-792.

15. Bubolz B, Case CL, McKay CA, O’Connor BK, Knick BJ, Gillette PC. Learning curve for radiofrequency catheter ablation in pediatrics at a single institution. Am Heart J 1996; 131:956-960.

16. Ho S, Accessory atrioventricular pathways: Getting to the origins. Circulation 2008; 117:1502-1504.

17. Misaki T, Watanabe G, Iwa T, Matsunaga Y, Ohotake H, Tsubota M, Takahashi M, et al. Surgical treatment of patients with WolffParkinson-White syndrome and associated acquired valvular heart disease. J Thorac Cardiovasc Surg 1994; 108:68-72.

18. Iturralde P, Guevara-Valdivia M, Rodriquez-Chavez L, Medeiros A, Colin L. Radiofrequency ablation of multiple accessory pathways. Europace 2002; 4:273-280.

19. Homoud M, Estes M, Wang P. Radiofrequency ablation of multiple accessory pathways. In: Huang S, (ed.), Radiofrequency Catheter Ablation of Cardiac Arrhythmias, 2nd ed. Armonk, NY, Futura Publishing, 2000, pp. 579-597.

20. Tanel RE, Walsh EP, Triedman JK, Epstein MR, Bergau DM, Saul JP. Five-year experience with radiofrequency catheter ablation: Implications for management of arrhythmias in pediatric and young adult patients. J Pediatr 1997; 131:878-887.

21. Hsu J, Tanel R, Lee B, Scheinman M, Badhwar J, Lee RJ, Tseng ZH, et al. Differences in accessory pathway location by sex and race. Heart Rhythm 2010; 7:52-56.

22. Bar-Cohen Y, Cecchin F, Alexander ME, Berul CI, Triedman JK, Walsh EP. Cryoablation for accessory pathways located near normal conduction tissues or within the coronary venous system in children and young adults. Heart Rhythm 2006; 3: 253-258.

23. Estes NA, III. Catheter cryoablation of supraventricular tachycardia: Quo vadis? Heart Rhythm 2004; 1:139-140.

24. Rodriguez LM, Leunissen J, Hoekstra A, Korteling BJ, Smeets JL, Timmermans C, Vos M, et al. Transvenous cold mapping and cryoablation of the AV node in dogs: Observations of chronic lesions and comparison to those obtained using radiofrequency ablation. J Cardiovasc Electrophysiol 1998; 9:1055-1061.

25. Khairy P, Chauvet P, Lehmann J, Lambert J, Macle L, Tanguay JF, Sirois MG, et al. Lower incidence of thrombus formation with cryoenergy versus radiofrequency catheter ablation. Circulation 2003; 107:2045-2050.

26. Zhou L, Keane D, Reed G, Ruskin J. Thromboembolic complications of cardiac radiofrequency catheter ablation: A review of the reported incidence, pathogenesis and current research directions. J Cardiovasc Electrophysiol 1999; 10:611-620. 